\title{
DE LA MORTIFICACIÓN A LA NEW AGE: GENEALOGÍA Y POLÍTICA DE LAS ESPIRITUALIDADES TERAPÉUTICAS CONTEMPORÁNEAS.
}

\author{
Mónica Cornejo Valle \\ Maribel Blázquez Rodríguez \\ Departamento de Antropología Social - Universidad Complutense de Madrid
}

http://dx.doi.org/10.5209/NOMA.53537

\begin{abstract}
Resumen.- Uno de los elementos centrales en la constitución de las espiritualidades contemporáneas es la progresiva incorporación de valores como la felicidad, el bienestar y el desarrollo personal en detrimento del rol espiritual del sufrimiento, el dolor físico y la mortificación. Como ha señalado Asad (2009) en su Genealogías de la Religión, tanto las morales seculares como la teología moderna han llegado a considerar el dolor y la tormento del cuerpo como signos de una espiritualidad arcaica y poco civilizada, en contraste con las teologías medievales, en las que precisamente la mortificación se consideraba una disciplina espiritual óptima. Ante ello nos preguntamos ¿cómo ha sido posible este giro radical? El objetivo de este artículo es dar cuenta del complejo haz de factores en cuya convergencia histórica se ha gestado la espiritualidad terapéutica contemporánea como un producto específico de la modernidad y su desenvolvimiento. Para ello, se tendrá en cuenta la relación entre religiosidad y bienestar/malestar, la asimilación del concepto de espiritualidad y las prácticas terapéuticas, y los diferentes contextos históricos en los que se ha ido gestando la autonomía del concepto de espiritualidad respecto al de religión.
\end{abstract}

Palabras clave: espiritualidad, New Age, salud, terapias alternativas, religión

\begin{abstract}
Wellbeing, happiness and personal growth have become central issues in contemporary spiritualties, despite of suffering, physiological pain or mortification. As Talal Asad (2009) has pointed out in his 'Genealogies of Religion', medieval theologies considered torment of the body and suffering in general as an optimum of spiritual discipline, while modern theology as well as secular morality agree to consider mortification and pain as archaic methods of uncivilized spirituality. Considering it, we wonder how this radical turn has been possible. The point of this paper is take into account the many and complex factors which converged to give birth contemporary therapeutic spirituality, while it is seen here as an historical result of Modernity. For that, we will explore the general relationship between religious experience and wellbeing/sickness, the convergence between therapeutic practices and spirituality understandings, and the historical contexts in which the notion of 'spirituality' gained autonomy from 'religious' realm.
\end{abstract}

Keywords: spirituality, New Age, health, alternative therapies, religion

Uno de los elementos centrales en la constitución de las espiritualidades contemporáneas es la progresiva incorporación de valores como la felicidad, el bienestar y el desarrollo personal en detrimento del rol espiritual del sufrimiento, el dolor físico y la mortificación. Como ha señalado Asad (2009) en su Genealogías de la Religión, tanto las morales seculares como la teología moderna han llegado a considerar el dolor y la tormento del cuerpo como signos de una espiritualidad arcaica y poco civilizada, en contraste con las 
teologías medievales, en las que precisamente la mortificación se consideraba una disciplina espiritual óptima tanto en las prácticas monacales como en las prácticas judiciales (en las que la tortura aparecía como un camino directo al encuentro con la verdad). Si bien es cierto que esta visión puede dicotomizar en exceso la religión moderna frente a la premoderna, e incluso oscurecer las duras disciplinas corporales que las sociedades misioneras modernas seguían reproduciendo en el siglo XIX (Comaroff y Comaroff 1991), no deja de ser cierto que ya hoy estas prácticas se conciben generalmente como algo espiritualmente improductivo, e incluso sádico. De hecho, en las prácticas judiciales actuales, el tormento del cuerpo se considera el tipo de práctica que hace a un grupo religioso sospechoso atentar contra la libertad de conciencia. Sin embargo, y bien al contrario de lo anterior, es más común que la espiritualidad se entienda hoy como una realidad íntimamente relacionada con la realización personal y el bienestar psicofisiológico. A veces se entiende como el camino a la felicidad y a veces como el resultado más pleno de los esfuerzos dedicados al crecimiento personal, pero ¿cómo ha sido posible este giro radical?

El objetivo de este artículo es dar cuenta del complejo haz de factores en cuya convergencia histórica se ha gestado la espiritualidad terapéutica contemporánea como un producto específico de la modernidad y su desenvolvimiento. Para ello, se tendrá en cuenta en primer lugar la relación entre religiosidad y bienestar/malestar, lo que se dibuja a través de la literatura tanto de la Antropología como de la Psicología de la Religión. En segundo lugar se tratará propiamente la asimilación del concepto de espiritualidad y las prácticas terapéuticas, empezando por esbozar los problemas de definición del concepto espiritualidad y siguiendo por la exploración de los diferentes contextos históricos en los que se ha ido gestando la autonomía del concepto de espiritualidad respecto al de religión. Por último, se analizarán algunas consecuencias de este devenir conceptual del tormento a la New Age, prestando especial atención a las dimensiones políticas del pensamiento positivo.

\section{LA RELACIÓN ENTRE EXPERIENCIA RELIGIOSA Y BIENESTAR/MALESTAR.}

Es necesario que el camino y subida hacia Dios sea un continuo cuidado de acallar $y$ mortificar los apetitos. Juan de la Cruz.

La literatura antropológica sobre la experiencia religiosa nos ha permitido comprobar en numerosas ocasiones cómo las cosmovisiones mágico-religiosas han proveído tradicionalmente a las poblaciones no sólo recursos y especialistas en el tratamiento del cuerpo y su bienestar/malestar sino de sus propias definiciones de enfermedad y salud ${ }^{1}$. A menudo estas concepciones

\footnotetext{
${ }^{1}$ Como se aprecia en manuales clásicos de Sociología de la salud, como el de Donati (1994), la teoría sociológica se ha preocupado más especialmente por las cuestiones relativas a la desigualdad, los aspectos institucionales de las prácticas en la atención a la salud y ciertas cuestiones definicionales.
} 
folk de la salud y del cuerpo se hallan vinculadas con diagnósticos morales de los síntomas, esto es, malestares concebidos y sufridos como expresión de desajustes respecto a las normas de convivencia, a los roles establecidos, a las conductas socialmente esperadas, a los ritos que deben cumplirse, etc. A pesar de las tempranas advertencias de Alexander A. Goldenweiser y Robert Lowie (1990:156), la mayor parte de la literatura clásica sobre este asunto establece la relación entre salud y religiosidad en sintonía con la noción durkheimiana de "eficacia simbólica". Quizá el ejemplo más conocido del enfoque funcionalista es, paradójicamente, uno de los antropólogos más antifuncionalistas, Claude Lévi-Strauss. En su popular artículo "La eficacia simbólica", Lévi-Strauss describe la cura chamánica como la manipulación psicológica (no física) del cuerpo enfermo (Lévi-Strauss 1995:216), poniendo en relación la parafernalia ritual chamánica con el psicoanálisis (Lévi-Strauss 1995:225). Así, si bien la dolencia que se trata tiene una manifestación somática, el malestar se identifica como un malestar social, y al identificar el origen social de la dolencia (diagnosticada en una parte de la escenificación ritual) el chamán viaja a los mundos míticos y a través de su trance induce una transformación orgánica (en otra parte de la escenificación). Un enfoque muy semejante lo podemos encontrar en la interpretación del chamanismo de Luc de Heusch (1973), donde aparece ya explícitamente el tema de la posesión. Y así también Alfred Metraux (1955) y Roger Bastide $(1961,1983)$ explican las virtudes curativas del trance en el vudú y en otras religiones afroamericanas en términos de eficacia simbólica.

Una variedad de este modelo interpretativo lo encontramos en los trabajos de Carmelo Lisón Tolosana sobre los endemoniados de la romería de El Corpiño en Galicia, donde volvemos a encontrar el tema del trance. Para Lisón, es la cultura la que "endemonia". Y la cultura, además, endemonia especialmente a las mujeres en la medida en que prevé para ellas una posición de debilidad estructural que se manifiesta a través de sus cuerpos y mentes en la forma de la posesión demoníaca. En este contexto, la celebración del exorcismo colectivo de El Corpiño representa no solamente un tratamiento cultural del síndrome sino también una explicación concreta, "al hacer la maldad localizable y explicable" (Lisón 2004:323). En sus trabajos sobre los cultos zar de Sudán, loan Lewis también planteaba un modelo de interpretación semejante cuando comparaba los ritos de exorcismo de hombres y los exorcismos de mujeres, concluyendo que los roles de género eran una función de la eficacia simbólica en el proceso ritual, determinando los roles tanto el tipo de malestar como el medio más apropiado para remediarlo. Y en esta línea, Pamela Constantinides (1977) ha llegado a descubrirnos los mismos ritos como una instrumentalización ocasionalmente deliberada por parte de las mujeres.

A pesar de la complejidad metodológica de su obra, el padre de la Antropología Médica actual, Ernesto de Martino, también estudió el tarantismo ${ }^{2}$ en un marco interpretativo en el que las relaciones entre religiosidad y bienestar/malestar se entendían de este modo durkheimiano, aunque no sólo. Aproximándose a lo que después llamaríamos "el trabajo de la cultura" con Obeyeskere (1990), De

\footnotetext{
${ }^{2}$ El tarantismo es el fenómeno cultural que asocia una crisis extática con la picadura de la tarántula, consistiendo la sanación en un tratamiento ritual con baile y música (tarantela, pizzicatta) y símbolos religiosos asociados especialmente a San Pablo.
} 
Martino apunta la idea de que los malestares culturales no tienen por qué coincidir con patologías físicas ni psicológicas reconocidas. Que no haya coincidencia entre los diagnósticos de la medicina y la psiquiatría no debe interpretarse como que la dolencia es fingida o voluntaria pues, como afirmaba también Lisón, la propia cultura dispone tanto las causas como las soluciones terapéuticas, y por lo general éstas soluciones terapéuticas en forma de ritos presentan una eficacia suficiente a la hora de devolver al sujeto a su "normalidad". En este sentido, podemos apreciar que toda la obra de De Martino está destinada a establecer tanto una perspectiva médicamente inteligible del tarantismo como a determinar los factores que unen en un solo fenómeno cultural los aspectos sociales, económicos, políticos, históricos, de género y los propios de la psicología individual que hacen manifestarse a las creencias religiosas en un cuerpo humano doliente. Sus conclusiones podrían extractarse así:

Hoy sabemos que el aguijón no es el ataque de un demonio o un dios, sino el pasado malo que vuelve y se somete a una reparación mundana. También sabemos que en el tarantismo la picadura o mordedura es un momento alienado de un remordimiento interior que se busca a sí mismo, "cierta pesadez u opresión interior", como decía Serao, el horizonte de una angustia que es el síntoma oculto de decisiones no tomadas y conflictos que obran en el inconsciente (De Martino, 1999:297-298)

Y por ello:

La tarántula, la picadura y el veneno tienen un significado simbólico en el tarantismo: liberan pulsiones inconscientes y las reacciones que suscitan en la conciencia individual. En este horizonte la tarántula, la picadura y el veneno entablan una serie de relaciones entre sí y con otras determinaciones, hasta formar un cuadro que posee su propia coherencia, siempre en el plano de la lógica simbólica. La tarántula, para desempeñar su función de símbolo, ante todo tiene que evocar y configurar, sacara a la luz y hacer revivir, los oscuros apremios del inconsciente que abruman la conciencia con su carácter oscuro e indomable" (De Martino, 1999:62)

Como se puede apreciar, la literatura antropológica que se ha preocupado por las relaciones entre experiencias religiosas y malestar/bienestar físicos se construye sobre un tipo de material etnográfico específico y emparentado: la fenomenología del éxtasis, y especialmente las variedades de la posesión, el trance y el chamanismo. Aunque estas tres categorías no describen lo mismo (y la discusión sobre su parentesco excede nuestros propósitos), lo cierto es que representan precisamente un campo de lo religioso caracterizado por su secular estigmatización, tanto en la medicina como, antes de eso, en el cristianismo. En este sentido, en algunas etnografías históricas nos llegan a describir aquellos contextos históricos y culturales en los que la propia manifestación religiosa llega a convertirse en una suerte de expresión social enferma. 


\section{1. ¿RELIGIOSIDADES ENFERMAS?}

Uno de los efectos de la progresiva hegemonía ganada por la biomedicina en la modernidad es, como sabemos, el repudio de las experiencias religiosas de manifestación corporal, y especialmente los trances. Ernesto De Martino caracteriza el rechazo de las experiencias extáticas por el Cristianismo y la medicina como las dos grandes polémicas de Occidente respecto a la relación entre salud y religión (1999:297), mostrando cómo la incomodidad de esta relación constituye un tema específico de la cultura mediterránea que posteriormente se convertiría en un tema que se expande a otras regiones como efecto de la colonización religiosa. $Y$, efectivamente, encontramos esto en las colonias de misión cristiana, como reflejan los trabajos citados antes de Roger Bastide y Alfred Metraux, en los territorios islamizados, como muestran los casos de loan Lewis y Pamela Constantinides, y también en la expansión budista, para lo que Gananath Obeyesekere (1985) ofreció las primeras referencias etnográficas, en particular, sobre la apatía mística y la depresión en Sri Lanka. De acuerdo con este panorama, podríamos estar de acuerdo con De Martino cuando afirma que "el tarantismo fue retrocediendo a enfermedad" a medida que la magia natural fue acusando la progresiva hegemonía de la Ilustración (1999:296).

En este sentido, la modernidad hace emerger una polémica cultural específica que ha caracterizado las tensas relaciones entre medicina y espiritualidad: el cuerpo "espiritado" como cuerpo enfermo y las expresiones corporales de la espiritualidad como expresiones sintomáticas de enfermedad mental. Como se puede ver, este tipo de caracterización es al mismo tiempo coherente con la forma en que desde el cristianismo se ha interpretado históricamente la experiencia extática como demoníaca o herética, esto es, como una patología espiritual sobre la que la iglesia ha desplegado sus propias políticas de higiene, prevención y sanación. Probablemente el mejor ejemplo de ello sean las bien documentadas historias de los propios místicos cristianos, que si bien hoy están santificados, en vida sufrieron una vigilancia severa de sus superiores eclesiales. También los documentos inquisitoriales son otra prueba elocuente de las tensiones morales que provocaban las formas de espiritualidad corporal no sujetas a la disciplina legítima de la época, cuando la manía de la posesión y el trance se quitaban con la mortificación de la carne.

Un ejemplo interesante de cómo la medicina toma el testigo de la religión institucional en el análisis y clasificación de estados físicos y mentales atípicos lo encontramos en uno de los trances colectivos más duraderos y polémicos de la historia religiosa europea: el caso de las visiones de Ezkioga. En su magnífica etnografía histórica, William Christian (1997) documenta referencias de los estados físicos de hasta 400 videntes y a partir de ello va describiendo la emergencia y evolución de un debate clave: si los las visiones, los estigmas y otros estados físicos y mentales atípicos eran prueba de lo sobrenatural, como los visionarios afirmaban, o si se trataba de un episodio particularmente descomunal de histeria colectiva (llegó a convocar hasta 80.000 asistentes en una sola noche). Sin embargo, aunque pudiera esperarse que de algún modo así fuera, lo significativo de este caso no es el alineamiento de sacerdotes por 
un lado y médicos por otro. Lo más interesante resulta el alineamiento de un puñado de religiosos y médicos partidarios de los visionarios por un lado, y su enfrentamiento a la condena constante del obispado (ratificada en 1934 por el Santo Oficio), de las instituciones psiquiátricas y el Estado (los videntes y sus partidarios fueron encarcelados en varias ocasiones y algunos retenidos en el psiquiátrico de Mondragón (Christian 1997:137). Todas las instituciones de la época (incluido ya también el periodismo) estuvieron de acuerdo en que las visiones de Ezkioga no eran sino un "maravillismo" insano (Christian 1997:401), como aseguraron varios psiquiatras y otros médicos, entre ellos algunos sacerdotes jesuitas que aparecen como figuras científicas expertas que clasifican las visiones al mismo tiempo de "procesos mentales puramente naturales" y carentes de características sobrenaturales (Laburu en Christian 1997:140). Aunque el episodio fue sumamente complejo, podemos advertir aquí el modo en que la Iglesia Católica cedía su propio terreno (lo sobrenatural) a la expertise positivista de la ciencia natural, siguiendo una cierta pauta de tradición religiosa y cultural según la cual los dominios del cuerpo no son los dominios de Dios. Más recientemente, esta tendencia ha ido cambiando.

\subsection{TRANSFORMACIONES EN EL CRISTIANISMO}

A pesar del rechazo conjunto de medicina y Cristianismo a ciertas formas de experiencia religiosa, en el episodio de Ezkioga ya no vemos la mortificación del cuerpo como remedio para el trance extático. De hecho, la evolución del Cristianismo en cuanto al papel de la espiritualidad en el bienestar psico-físico ha seguido la evolución que en la propia medicina se ha dado desde una medicina ocupada en la curación de la enfermedad a una medicina centrada en el concepto de salud (y en la prevención). De este modo, y aunque los medios cristianos acusan a la New Age de constituir apenas una desviación hedonista de una espiritualidad más profunda, lo cierto es que el desplazamiento de una espiritualidad del tormento a una del bienestar no es extraño tampoco al Cristianismo contemporáneo. Así, se ha pasado de una teodicea de la enfermedad encardinada en la reflexión general sobre el sufrimiento, hasta una Teología de la salud orientada a la promoción del autocuidado y del cuidado de los otros, en una lógica de respeto a la vida.

Si bien es cierto que el acompañamiento de enfermos es una labor cristiana siglos antes de la llegada de la propia ideología del bienestar (al menos claramente desde la formación de la Orden de los Ministros de los Enfermos, o los hermanos camilos, en 1582) y que el proceso de implantación de la clínica en Europa no podría pensarse sin la presencia de religiosas y religiosos en los hospitales, en los años sesenta del siglo $X X$ se producen algunos cambios relevantes. En el caso del Catolicismo, los cambios se aprecian en las diferentes actitudes que presentan Pablo VI en la encíclica Humanae Vitae, y después Juan Pablo II en Evangelium Vitae. Aunque estos textos se hayan hecho más conocidos por las polémicas alrededor del aborto, la eutanasia o las células madre, el vitalismo que predican ha servido también de fundamento a la llamada Teología de la salud. 
En su momento, un primer impacto de esta apertura espiritual a la vida en sus formas corporales se da en la corriente de la Teología de la Liberación católica. El que fuera el movimiento católico más importante en las décadas de los setenta y ochenta del siglo $\mathrm{XX}$, hace del rechazo al sufrimiento, en todas sus formas, una bandera, como sostiene Leonardo Boff (1985). Sin embargo, no deja de ser significativo que a día de hoy la revista del Consejo Pontifico para la Pastoral de la Salud lleve por título Dolentium Hominum, y que las jornadas mundiales del enfermo no hayan cambiado su nombre por alguno más próximo a la promoción de la salud. Como se aprecia en los resúmenes históricos de la revista, la línea general de la pastoral sigue fuertemente anclada a una cierta hegemonía del padecimiento en la que la espiritualidad se concibe como una dimensión positiva para la vivencia de la enfermedad, en la línea actual de la psicología de la religión. Más allá de esto, una vía relativamente nueva de apertura hacia la espiritualidad terapéutica en el Catolicismo es la que procede, aunque todavía tímidamente, de las corrientes del pentecostalismo y la Renovación Carismática (Gower 2006).

En el caso de la espiritualidad pentecostal, tanto católica como protestante, la transformación que se percibe no es tanto teológica como práctica, pues en ambos casos estamos ante formas de religiosidad que admiten la posesión divina (por el Espíritu Santo) y con ella se asocian diversas formas de sanación mediante la imposición ritual de manos y la encarnación de carismas (Cornejo 2001). Estas prácticas han atraído la atención de numerosos antropólogos y sociólogos, tanto especialistas en religión como en salud, pero los primeros trabajos de relevancia se los debemos a Meredicth McGuire, quien establece algunas interpretaciones sobre las que hoy sigue habiendo cierto consenso. Así, en su "Ritual Healing in suburban America" (McGuire 1988), la autora aborda directamente la cuestión de la sanación como uno de los elementos clave que el conjunto de los Nuevos Movimientos Religiosos habían introducido en la década de los setenta del siglo XX. En este caso, y a diferencia de otros fenómenos investigados desde la antropología, McGuire destaca que no estamos ya ante unos sistemas de creencias más o menos premodernos que resisten como pueden a la modernidad, sino más bien un fenómeno particular de revitalización, de adaptación a la modernidad, un fenómeno religioso específicamente postmoderno que desde su surgimiento (en la década de los setenta del siglo $\mathrm{XX}$ ) reivindica la salud como dominio de la espiritualidad, constituyendo.

Para abordar el tema, McGuire hace una muestra de 130 grupos y sanadores diferentes, algunos de los cuales fueron estudiados intensivamente con observación participante de más de 10 meses, otros con trabajos de campo más esporádicos, con hasta un total de 255 sesiones grupales de 31 grupos distintos observados y grabados con detalle. También 330 entrevistas y un grupo de control de 43 personas (McGuire 1988:9). Uno de los elementos más interesantes de este trabajo es que la muestra incluye sanadores y grupos alternativos tanto cristianos como "metafísicos" y humanistas (New Age), lo que permite compararlos y dar un paso adelante en nuestra perspectiva sobre la evolución de esta convergencia entre salud y espiritualidad. Gracias a esta estrategia, McGuire identifica una cuestión clave que aquí hemos llamado "el trabajo de cultura" (Obeyeskere 1985): aunque los estudios sobre salud y 
sanación alternativas asumen que los practicantes de tal sistema alternativo comparten las nociones de enfermedad y sanación propias de los sistemas médicos, esto no es así, la definición de lo que es salud y enfermedad parte de su propio esquema de planteamiento, de modo que tanto las manifestaciones físicas como psicológicas de las dolencias, así como también sus causas, para las que encuentran tratamiento en los ritos vienen dadas por la propia creencia religiosa (McGuire 1988:32-33). De hecho, McGuire encontró que sólo unos pocos creyentes o practicantes de la sanación alternativa se acercaron a los grupos por problemas específicos de salud o por una curiosidad asociada. Antes al contrario, la mayoría se adhieren a los grupos y filosofías que practican la sanación alternativa por interés en sus sistemas de creencias, no por sus prácticas de sanación, lo que de nuevo nos pone ante diagnósticos morales sobre los malestares del cuerpo.

Como estableció McGuire (y otros muchos especialistas del pentecostalismo han ido asumiendo), el advenimiento de la espiritualidad pentecostal y su particular dimensión terapéutica constituyen una consecuencia compleja de las transformaciones generales en el rol que el individuo moderno ha ido sufriendo en las sociedades contemporáneas. La autora llama la atención sobre cómo la enfermedad, el malestar, el cuidado y la sanación afectan profundamente a la identidad y al sentido de uno mismo. En esta línea, el individualismo resultante del énfasis en la auto-sanación y en el frecuentar las terapias alternativas estaría intentando adaptar al individuo y a la salud a los imperativos político y económicos actuales de flexibilidad social, constituyendo específicamente dispositivos culturales de adaptación (por la espiritualidad) a un modelo de sociedad en el que constantemente se cambia de roles y de vínculos y en el que las experiencias emocionales y físicas ganan centralidad (McGuire 1988:238). Así, los enfoques de salud alternativos no irían en contra de la práctica biomédica sólo por su énfasis en el holismo sino también en la medida en que reubican el lugar del individuo en el proceso de sanación, sino que constituirían una acción afirmativa contra la racionalización del cuerpo y las emociones que se preveían en la modernidad como consecuencia de la secularización (McGuire 1988:240).

\subsection{LA EVIDENCIA CLINICA ENTRE PUGNAS}

Uno de los datos más importantes a la hora de establecer cómo han evolucionado las relaciones entre espiritualidad y bienestar/malestar se aprecia también en la literatura médica que da cuenta de ello, donde encontramos, entre otras cosas una de las revistas más antiguas sobre el tema (The Jornal of Religion and Health, editada por Springer desde 1961). Para empezar, como ha señalado Harold G. Koenig (2008), uno de los más relevantes especialistas en el tema, esta literatura ha visto un repentino crecimiento en las últimas décadas. Antes del año 2000 se podían contar más de mil investigaciones con resultados publicados en las que se examinaban cuantitativamente las relaciones entre religión/espiritualidad por un lado y salud por otro. A mediados de 2010, ya había al menos 2000 estudios cuantitativos más que han dado lugar a unos 3000 informes originales de investigación empírica sobre la asociación entre religión/espiritualidad y salud mental, salud física y uso de los 
servicios sociales (Koenig, King and Carson 2012:461). Como señalan Quinceno y Vinaccia (2009), los estudios se pueden clasificar entre aquellos centrados en la salud física y los centrados en salud mental. En cuanto a la salud física los estudios han estado centrados particularmente en la actividad inmunológica, las neoplasias y enfermedades cardiovasculares, cerebrovasculares, neurológicas y dolor, por un lado, y en cuanto a la salud mental, por otro lado, los estudios han estado enfocados en las adicciones, el suicidio, la delincuencia, la ansiedad-depresión, el estrés, la esquizofrenia, el psicoticismo y los trastornos bipolares. $Y$ en todos estos trabajos parece que obtiene como resultado un mismo principio: relaciones positivas y causales concluyentes entre espiritualidad y salud.

En general, estos estudios se alinean en el enfoque biopsicosocial que caracteriza lo religioso como una suerte de dispositivo multidimensional de carácter cognitivo-afectivo-conductual, y no tanto como una condición sui generis, con una naturaleza (espiritual) propia (algo que sólo se aprecia y reivindica en la literatura de creyentes, que si bien es números, constituye una minoría del conjunto en la literatura médica, Park et al. 2011, Quinceno 2009). A pesar de que este enfoque tiene en cuenta los factores sociales de las creencias y prácticas religiosas, lo cierto es que desde los años sesenta, las investigaciones en este campo muestran un cierto optimismo característico que procura llamar la atención de los médicos hacia los efectos positivos de considerar lo religioso como un factor activo y eficaz en la intervención en salud, especialmente en la medida en que la mayor parte de las investigaciones convergen en una misma conclusión reiterada: los individuos que carecen de motivaciones de tipo religioso presentan una menor motivación en el cuidado de su salud. No obstante, en las investigaciones nunca aparecen las motivaciones, modelos o conductas religiosas como dispositivos que desencadenan patologías por sí solos.

A pesar de la reiteración de los resultados, el avance en la investigación se ha está algo estancado. Jeff Levin (2009), conocido por su propuesta de una teosomática médica, explica este estancamiento por el escaso impacto que estas investigaciones están teniendo en nuestra comprensión de la salud y de la sanación, y ello se debe en buena medida al antagonismo entre dos posturas radicalizadas: la de los partidarios de una medicina holística y espiritual que acusan de arrogante al modelo biomédico pero dejan ver excesos de confianza respecto a los resultados de tales técnicas holísticas y espirituales, y la de los defensores a ultranza del modelo biomédico, algunos de los cuales creen no haber sido lo bastante estrictos en la apología de la biomedicina a juzgar por las bizarras alternativas que se proponen desde algunas corrientes de la New Age. Según Levin, ambas posturas están exagerando y defendiéndose por demás, en detrimento de aportaciones y reflexiones más reposadas y serias, pero incluso desde la teología se ha expresado una cierta preocupación sobre la posible instrumentalización de la religión para fines no espirituales como el mantenimiento o la mejora de la salud (Shuman y Meador 2003).

Por si este combate en el seno de la investigación médica no fuera bastante, los medios de comunicación han resultado pobres aliados de la difusión científica, caricaturizando los hallazgos sobre espiritualidad y salud con titulares 
sensacionalistas que confunden sobre las implicaciones de las investigaciones. Harold Koenig (2012) explora dos ejemplos. En un caso, se trata de los resultados de un estudio clínico sobre la implicación religiosa y las características de la memoria (en particular, el tamaño del hipocampo) en la que los protestantes "renacidos" (principalmente pentecostales) presentaban una morfología del hipocampo ligeramente distinta a los protestantes históricos (o fríos). El principal periódico protestante del país, Christian Century publicó la noticia con el siguiente título: "Un estudio sugiere que los creyentes renacidos tienen cerebros más pequeños". Obviamente, estas actitudes no ayudan a mostrar la pertinencia ni la importancia de los hallazgos científicos. Algo parecido denuncia Koenig sobre el New York Times. Una importante investigación sobre religiosidad y autocontrol/autorregulación pretendía contrastar si la espiritualidad personal de los individuos guardaba alguna relación con las conductas de autorregulación y si esto a su vez tenía algún efecto en dos ámbitos: la salud de los sujetos y la conducta antisocial. El 30 de diciembre de 2008 The New York Times presenta la noticia afirmando. Jocosamente, que los lectores deben ir a la iglesia o correrán el riesgo de convertirse en criminales o empeorar su salud. Sin duda, manipulaciones y caricaturas como éstas no contribuyen a la profundización sobre la materia.

Pese a todo, hay dos desarrollos más relevantes en el campo que constituyen aportaciones sustantivas: las taxonomías de la espiritualidad realizadas desde una perspectiva médica (generalmente desde la psiquiatría), y las herramientas técnicas para medir la espiritualidad (cuestionarios e imagen cerebral). Respecto a la medición de la espiritualidad, esta literatura médica aporta un punto de vista original que no se encuentra en las ciencias sociales, empezando por el hecho de que desde hace algún tiempo se viene empleando el concepto espiritualidad, mientras los cuestionarios sociológicos siguen empleando generalmente el de religión (Hall, Meador and Koenig 2008). Así también, en Psicología y Psiquiatría se adopta un punto de vista centrado en el individuo, mientras que la Sociología se centra en cuestiones como la adscripción religiosa. Y mientras la Sociología se ha preocupado de la métrica de la adhesión y la participación de los fieles, los cuestionarios de la Psicología y la Psiquiatría han venido intentando la aprehensión cuantitativa de la experiencia espiritual vivida, incluyendo las emociones y las sensaciones corporales. Gracias a estas diferencias, la información cuantitativa más relevante sobre espiritualidad procede hoy de la Psicología de la religión.

En parte como consecuencia de los avances en la medición de la espiritualidad, en esta literatura encontramos interesantes discusiones sobre las tipologías de la espiritualidad que dan lugar tanto a tipologías graduales (más religioso, no religioso) como a clasificaciones cualitativas, como la que usan Koenig (2008) o Levin (Levin, Taylor and Chatters 1994). Mientras la de Levin se articula en función el grade de institucionalización de la práctica espiritual, la de Koenig articula varios factores complejos, facilitando poner en perspectiva las evoluciones recientes de la espiritualidad contemporánea. De hecho, de forma bastante novedosa y acertada, Koenig no identifica la ausencia de práctica espiritual con el ateísmo o la ausencia de ideas religiosas (como hacen la mayoría de cuestionarios), sino que la interpreta como una 
forma particular de concebir el cuerpo y el bienestar. Los cuatro modelos de espiritualidad que propone son los siguientes:

(1) Versión tradicional-histórica de Espiritualidad. Coincidiendo con un concepto convencional de religión, se caracteriza por una religiosidad donde la autoridad de los pastores o los sacerdotes es fuerte para el creyente y la tasa de compromiso comunitario alta, con intensa participación y asistencia al culto. La espiritualidad en esta versión sería completamente autónoma respecto al cuerpo y la salud mental o física.

(2) Versión moderna de espiritualidad. Se caracteriza porque se aplica a "personas espirituales, pero no religiosas". Esta versión conceptualiza la comparación de la salud mental y física de los que son "espirituales religiosos", los que son "espirituales, pero no religiosos", y los que son "completamente seculares", de tal manera que en cuestión de salud econtramos irregularidades características: para unos la espiritualidad forma parte de su cuidado y para otros no.

(3) Versión tautológica moderna de espiritualidad. Similar a la anterior en su consideración de la espiritualidad pero extendida hacia cuestiones como el desarrollo personal, el crecimiento interior, la autorrealización existencial (el programa ideológico del Movimiento del Potencial Humano). En este sentido, lo espiritual no consiste en aspectos necesariamente sagrados sino, sobretodo, en estados psicológicos positivos, significado de la vida, conexión con los demás, paz interior, armonía, bienestar y otros elementos ya influidos por la New Age. Koenig usa el término "tautológico" para llamar la atención sobre una retroalimentación específica de este tipo de espiritualidad humanística con el estado de bienestar, pues en cierto modo, en este modelo el cultivo de la espiritualidad equivale al cultivo de la propia salud, entendida como bienestar y armonía. En este modelo, las personas que se autodefinen como no espirituales continúan considerando por separado la salud y las creencias.

(4) Versión clínica moderna de espiritualidad. Este modelo es una extensión integracionista del anterior que más que representar una situación parece que el propio Koenig propone como modelo de posible implantación. Se caracteriza por incluir a las personas que se autodefinen como no espirituales como una forma más de espiritualidad, de manera que su particular religiosidad sea considerada desde la clínica como un elemento más a tener en cuenta, con independencia de si el paciente lo encuentra pertinente o no.

A la vista de esta tipología, así como del resto de aspectos y contextos en los que se vienen dibujando las relaciones generales entre la experiencia religiosa y la cuestión del bienestar/malestar, resulta evidente que está faltando explicitar acerca de qué se habla al hablar de espiritualidad. En efecto, se habla de varias cosas, emparentadas aunque distintas, $y$, en todo caso, sujetas a numerosas pugnas definicionales que no dejan de reflejar la genealogía política de los conceptos con los que pensamos la realidad social y sus transformaciones. 


\section{GENEALOGÍA POLÍTICA DEL CONCEPTO “ESPIRITUALIDAD”}

Hay especies de verdades que desaparecen con el mundo moral, como hay razas de animales que perecen en el mundo físico. Chateaubriand.

Aunque aquí asumimos que el concepto de espiritualidad contemporáneo tiene una historia política concreta (que intentamos explorar), lo cierto es que una gran parte de la literatura contemporánea sobre el tema considera la espiritualidad es una suerte de impulso universal y ahistórico en el ser humano, en particular el que explicaría la actual posibilidad de declararse "espiritual pero no religioso" (Heelas y Woodhead 2008, Flanagan y Jupp 2007, Giordan 2007, Forman 2004, Roof 2003). Esta perspectiva es compartida también por investigadores médicos como Koenig, Levin y otros. De hecho, en la literatura anterior destacan dos elementos de definición de la espiritualidad: (1) la diferencia respecto a la religión, en concreto, la espiritualidad comparece como la variación individual y subjetiva de aquello que hacen y son las religiones, y (2), si las religiones son respuestas históricas a la búsqueda de sentido, religación y trascendencia, la espiritualidad aparece aquí como la versión presocial de la religión, y en este sentido como una suerte de impulso panhumano y eterno en pos de tales religación y trascendencia (George, Larson, Koenig y McCullough 2000, Quiceno y Vinaccia 2009).

Por lo que respecta a las ciencias sociales, donde el punto de vista de los actores sociales se puede instituir en guía definicional, la exploración de definiciones se ve inmediatamente hostigada por uno de los conflictos habituales en la investigación social sobre creencias: las necesidades teóricas de definición de un fenómeno cultural no encajan (y en virtud de su capacidad heurística, no tienen que encajar forzosamente) con las necesidades de definición de los creyentes, aunque las definiciones de estos creyentes sean el fenómeno cultural mismo. En Antropología, este debate no es más que una extensión peculiar de los problemas epistemológicos que etiquetamos a través de la dicotomía emic-etic, y a menudo el problema se resuelve de forma episódica, por decir así, y en función de las prioridades de la investigación. De este modo, en algunos momentos de la investigación es prioritario dar apropiada cuenta de las definiciones que ofrecen los actores sociales, y en otros momentos es relevante ofrecer definiciones técnicas que den cuenta de la diversidad de puntos de vista (sin ajustarse a ninguno de ellos en particular). No obstante, el problema no ha de terminar aquí, pues la diversidad de las propias definiciones técnicas llega a poner en juego las premisas ontológicas que los propios investigadores introducen en la ecuación. Por lo que respecta a la discusión sobre la espiritualidad, no existe (y es posible que no llegue a existir) un consenso respecto a si la espiritualidad es una o son muchas.

Aunque los estudios contemporáneos sobre la espiritualidad desde las Ciencias Sociales no hayan pretendido participar en los debates teológicos y filosóficos sobre la naturaleza ontológica de la espiritualidad, las posturas han permanecido igualmente divididas. Como señala Peter Holmes en su introducción al clásico contemporáneo de Flanagan y Jupp "Sociology of 
Spirituality", la espiritualidad puede entenderse de forma genérica como la búsqueda relacional de significado del ser humano, lo que "para muchos esto hoy incorpora una dimensión sobrenatural o sobrecorpórea que sugiere que muchos de nosotros hemos descubierto que somos más que nuestra biología física" (Flanagan y Jupp 2004:25). Sin embargo, la generosa pretensión de Holmes por abarcar en su definición el mayor número posible de variaciones culturales de la espiritualidad no deja de ocultar la relevancia de estas variaciones históricas, incluyendo al menos dos elementos: un desplazamiento del concepto respecto a las definiciones tradicionales de las religiones abrahámicas (Cristianismo, Islam, Judaismo), para las que no existe una espiritualidad sin Dios, y el contexto histórico en el que este desplazamiento tiene lugar, que incluye la adopción de formas orientalistas (no necesariamente orientales) de concebir lo espiritual.

Respecto al desplazamiento desde la espiritualidad monoteísta tradicional a la contemporánea, cabe señalar que la definición de Holmes peca del mismo etnocentrismo que Talal Asad nos hiciera ver en la archiconocida definición de Clifford Geertz de religión (1995:89). La importancia que Geertz (siguiendo a Weber) daba a la búsqueda de significado en las religiones ignora por completo dos cosas: primera, que los significados se han impuesto históricamente, que tienen sus genealogías, sus conflictos, sus defensores y sus perseguidores; y, segunda, que esta idea de la religión como búsqueda, aunque podría aplicarse a ciertos individuos singulares (místicos, reformadores y otros cuyas biografías recogen también abundantes conflictos), olvida las prácticas históricas de imposición de una verdad revelada, donde la búsqueda no era en absoluto la dimensión relevante de la religión. Ante este tipo de dificultad, como recomienda Asad, cabe preguntarse no sólo qué es la espiritualidad sino cómo ha llegado la espiritualidad a definirse en relación a las búsquedas de significado. Así mismo, Talal Asad también invita a tener en cuenta (2009:12) que algunas prácticas de espiritualidad tradicionales como la mortificación del cuerpo (más o menos hegemónicas en varias de las religiones mundiales en distintos periodos), no coinciden con las formas dominantes de la espiritualidad en la religión moderna y menos aún en la espiritualidad contemporánea, donde justamente encontramos la tendencia contraria.

Para Robert Forman (2004), la mejor forma de afrontar este dilema definicional es prestar atención a los usos que diferentes informantes ofrecen del concepto de espiritualidad, tal y como aparece en las entrevistas y en la observación participante. En Grassroots Spirituality, Forman recoge cuatro usos distintos del concepto contemporáneo de espiritualidad: (1) Por un lado, reconoce un uso general y difuso que incluye referencias a las siguientes expresiones: trabajo interior, meditación, quietud, apertura, alivio. En ocasiones, esta noción de espiritualidad presenta un matiz introvertido, centrado en la conciencia de uno mismo, en el vacío mental místico. Otras veces presenta un matiz relacional que se expresa como "resonancias, campos magnéticos entre cosas", "conexiones" que pueden darse entre objetos, personas, situaciones, etc. Esta forma de presentar el significado del concepto facilita, además, una definición emic de tipo ostensivo. (2) Por otro lado, hay una noción de espiritualidad que claramente se define en oposición binaria a otras categorías, como las de dogma, doctrina, institución, obligaciones, religión, cristianismo; (3) También 
resulta significativo para Forman el sentido etimológico de spirit como respiración o viento, aliento, aliento vital, lo que en otras lenguas sería equivalente a palabras como ruah, pneuma, atman; (4) Por último, Forman ofrece una definición operativa que probablemente sea la más conocida de su trabajo, definición conforme a la cual la espiritualidad en su investigación ha emergido como:

"una ultimidad vagamente panenteísta que es habitada, a veces corporalmente, como el más profundo sentido de uno mismo, y a la que se accede a través de medios no estrictamente racionales de autotransformación así como a través de procesos grupales que convierten la vida en una organización holística para todo" (Forman 2004:51).

Esta secuencia de cuatro definiciones permite comparar al mismo nivel de relevancia teórica las definiciones múltiples de los actores sociales sobre la experiencia espiritual y aquellas definiciones técnicas que pueden proponerse como unitarias, permitiendo manejar un concepto operativo útil para dar cuenta de la diversidad interna en lo que se define. A pesar de ello, lo que se desprende de las definiciones de Forman es claramente un concepto de espiritualidad marcado por la experiencia subjetiva (no por una doctrina), aunque ésta se construya discursivamente en referencia a algunos conceptos globales presentes en la filosofía New Age (transformación interior, conexión, etc.). Sin embargo, siguiendo aquella recomendación de Talal Asad, se hace imprescindible revisar cómo surge y de dónde surge este concepto individualista y New Age de espiritualidad que hoy en día se ha extendido, llegando a impregnar también (no siempre con éxito de crítica) las prácticas cristianas y otras.

\subsection{EL ESPIRITISMO ES SECULARISMO}

Como ha señalado Peter Van Der Veer (2009), uno de los efectos más interesantes del advenimiento del concepto contemporáneo de espiritualidad es precisamente la idea de que es una y múltiple, sin que ello implique la contradicción (o incluso la herejía) que en otros momentos o contextos puede implicar. Esta idea característica de la espiritualidad universalista contemporánea, que obviamente pivota sobre la constitución del sujeto moderno occidental y las ideologías individualistas que lo relatan, se ha gestado, según Van Der Veer en dos fuentes clave: el secularismo y el orientalismo. Por lo que respecta a la fuente secularista, Van de Veer llama la atención sobre la sensibilidad esotérica de algunos de los más destacados líderes del librepensamiento, entre ellos el agnóstico George J. Holyoake (conocido precisamente por haber inventado el término "secularismo") o el ateo fundador de la National Secular Society, Charles Bradlaugh. Quizá el ejemplo más relevante de esta coincidencia es el de Annie Besant, amiga personal de Bradlaugh y tan conocida por ser una de las iniciadoras del feminismo contemporáneo (en el que la crítica al Cristianismo cumplió un papel esencial) como por presidir la Sociedad Teosófica fundada por Madame Blavatsky, sus actividades en la Co-masonería o por ser co-fundadora de la Orden del Templo de la Rosacruz en Londres. También el padre del ocultismo contemporáneo, 
Eliphas Lévi (Alfred Charles Constant), destacó profesionalmente como periodista de izquierdas y difusor del anarquismo, siendo su "Dogma y ritual de la Alta Magia" un hito de la heterodoxia intelectual y religiosa (Horta 2001:190), además de un sólido exponente de la creencia en una única espiritualidad panhumana reprimida por las miserias de la iglesia.

Como se desprende de lo anterior, la convergencia de una sensibilidad simultáneamente secularista y espiritual ya entonces guardaba relación con la oposición a las formas religiosas históricamente impuestas por la alianza entre Iglesia y Estado, que es uno de los elementos clave en la definición contemporánea de lo espiritual (Cornejo 2012, Forman 2004). Y ello no era únicamente una cuestión de acusaciones cruzadas en libros y artículos. De hecho, Besant y Bradlaugh fueron acusados conjuntamente del delito de blasfemia por presentar principios ateístas en la publicación de un panfleto que defendía el uso de métodos anticonceptivos (Besant 2011:213). Coincidencias semejantes las encontramos en la convergencia de espiritualidad y racionalismo de otros influyentes pensadores de la época, como el del alemán Karl C.F. Krause, quien fue, precisamente, una figura de gran influencia en los países de habla hispana, siendo el referente intelectual de la Institución Libre de Enseñanza en España o de la Unión Cívica Radical en Argentina. Entre los krausistas españoles, además de la fuerte influencia intelectual y mística del propio Krause, se extendió especialmente la doctrina de Allan Kardec (Hippolyte Léon Denizard Rivail), fundador de la corriente espiritista alternativa a la Teosofía. Según Lujambio, la influencia "espírita" se empezó a fundir con el krausismo después de que en 1861, el Obispo de Barcelona ordenara la destrucción pública de trescientos libros que el propio Kardec había enviado a un librero de Barcelona (Lujambio 2011:131). También es conocido que uno de los libros de cabecera del fundador del Partido Socialista Obrero Español, Pablo Iglesias Possé, no era otro que "El libro de los espíritus" de Allan Kardec (De Motes Bermet 1977:197). Sin embargo, si bien Pablo Iglesias Mossé representaba una facción del socialismo contraria al anticlericalismo, otras facciones muy activas en la crítica al Catolicismo en España provenían precisamente de la masonería (Álvarez Lázaro 2006:254), mostrándonos otra vez el vínculo entre el desarrollo del pensamiento secularista y el nacimiento de un nuevo concepto de espiritualidad que tendía al universalismo precisamente basándose en la crítica a la religiosidad eclesial.

Como se puede apreciar en las referencias anteriores, el concepto contemporáneo de espiritualidad estaba en su origen comprometido con las peculiaridades políticas de su tiempo y no sólo con algún tipo de evolución espiritual histórica o ideológicamente autónoma. Así también, la otra influencia fundamental que Van Der Veer destaca en la constitución del concepto contemporáneo de espiritualidad, la influencia orientalista, no estará menos vinculada con la dimensión política. $Y$ es aún más claramente en el orientalismo donde encontramos la tendencia a concebir la espiritualidad como una realidad universal y panhumana. De una forma general, esta tendencia nace del interés en establecer un diálogo pacífico entre dos universos conceptuales tan diferentes como el oriental y el occidental. 


\subsection{LA ESPIRTIUALIZACIÓN DE ORIENTE}

Este interés por el diálogo interreligioso pacífico ya se aprecia en el cristianismo colonial del siglo XIX, con antecedentes en los debates de los siglos XVI y XVII sobre la naturaleza espiritual de los indios en las colonias americanas del Reino de España. Sin embargo, la dimensión política más evidente de este esfuerzo se encuentra en las vicisitudes del primer Parlamento de las Religiones, celebrado en Chicago en 1893. La propia organización de este evento, que dio nacimiento entre otras cosas a la expresión "religiones mundiales", suponía la aceptación de tradiciones religiosas no cristianas en pie de igualdad moral, y constituye un hito del pluralismo religioso contemporáneo en la medida en que escenifica por primera vez el reconocimiento de la legitimidad de otras verdades reveladas y otras formas de espiritualidad y relación con lo sagrado. No obstante, el evento no careció de polémicas a la hora de decidir cuáles serían las religiones legítimas y cuáles no. Las religiones naturalistas, animistas, tribales, etc. no tuvieron ninguna presencia en parlamento, y la desproporción entre denominaciones protestantes y las islámicas (reducidas a una) era sintomática de la forma fuertemente cristianocéntrica de concebir esa espiritualidad universal que se pretendía compartir. La Iglesia Católica rehusó participar en aquella primera edición del Parlamento, escandalizada por la pretensión de los organizadores de colocar al representante de Pedro entre quienes consideraba herejes y paganos. Sin embargo, la inclusión de una religión no deísta como el Budismo, representó muy eficazmente ese intento por cultivar el diálogo de las espiritualidades humanas diversas en una unidad negociada y escenificada.

Al mismo tiempo, esos intentos por aislar elementos generales de espiritualidad en las tradiciones religiosas existentes dependieron también del desarrollo de las nuevas herramientas filológicas y lingüísticas que permitían analizar las tradiciones religiosas y traducir los textos chinos e indios principalmente, en los que no existen términos equivalentes a la noción occidental de espiritualidad (aunque sí hay palabras para el concepto de espíritu). El antropólogo y lingüista Max Müller fue uno de los principales responsables de lo anterior gracias a la realización de la primera traducción a la lengua inglesa del Rig Veda, y gracias también a sus trabajos sobre confucianismo y taoísmo, que en su obra son consideradas religiones mundiales. Así también, sus trabajos como lingüista tuvieron una influencia relevante en el pensamiento antropológico gracias a su forma particular de entender algunos conceptos clave de la Antropología del Religión, como los de mana, orenda, etc. Sin embargo, uno de los efectos más interesantes de todo este proceso característico de la emergencia del orientalismo va más allá de los esfuerzos occidentales por "entender" oriente y consiste en el uso estratégico de este orientalismo por parte de las sociedades orientales, y especialmente los estados de India y China.

Como señala Van der Veer, el concepto de espiritualidad ha jugado un rol crucial en la historia contemporánea de los estados asiáticos y sus discursos nacionalistas. En la India se adoptó ampliamente la visión orientalista propia del romanticismo europeo en la medida en que esa visión celebraba la civilización hindú como una cultura altamente apreciada por sus cualidades espirituales, lo que tuvo un papel relevante en el proceso de independencia nacional. Entre los 
representantes del espiritualismo nacionalista hindú estaba Swami Vivekananda, que fue el representante del Hinduismo en el Parlamento de las Religiones de Chicago y que hoy se reconoce como el primer promotor del yoga en Estados Unidos y la inspiración de las primeras Sociedades Vedanta americanas. Su espiritualidad estaba lejos de ser pacífica y tranquila. Criticaba el arrobamiento místico y la renuncia espiritual al mundo, y defendía posiciones más activas de los indios frente a los poderes coloniales. Sin embargo, Vivekananda realiza la transición entre el hinduismo que huye del mundo y el que se involucra en él introduciendo elementos de pacifismo y tolerancia en su discurso, no incompatibles con la provocación, las demandas de libertad y el orgullo nacionalista (Borreguero 2004).

La fusión del nuevo espiritualismo hindú con el nacionalismo político dio lugar en primer lugar a un acoplamiento de la visión que los occidentales tenían de la espiritualidad. Este acoplamiento se aprecia en el propio Vivekananda (fuertemente criticado por sus coetáneos por haberse occidentalizado en la medida en que su visión era crítica con el tradicional rechazo del mundo del misticismo hindú), pero también en movimientos de reforma local sobre los que Vivekananda influyó directamente (como el movimiento Brahmo Samaj liderado por Raja Rammohun Roy), y en otros posteriores, como Ananda Marga, Meditación Trascendental o Hare Krishna, que se cuentan entre los más conocidos en Europa y Estados Unidos. Y así también, este nuevo discurso de la espiritualidad volcada al mundo tuvo un fuerte impacto en figuras como Gandhi o Tagore, que hicieron de esa espiritualidad un recurso político aunque hoy en día se citan como representantes de una espiritualidad ahistórica. Ambos son representantes ampliamente conocidos de un discurso según el cual el materialismo de Occidente crea guerras y explotación colonial mientras que la espiritualidad de Oriente propondría una alternativa de paz y prosperidad para todos.

Por lo que respecta a la figura de Gandhi, éste se encuentra característicamente entre los personajes históricos que destacan por una notable aureola religiosa en las representaciones occidentales. Sin embargo, Van der Veer contrasta el uso político que Gandhi hizo de la espiritualidad con el más íntimo sentido espiritual de Nehru, que sin embargo es reconocido en occidente sólo como un reformador político. Según Van der Veer, Gandhi adoptó el discurso del espiritualismo universalista, afín a los postulados de Vivekananda y del Parlamento de las Religiones instrumentalizando la cuestión espiritual de manera que el nacionalismo indio no sufriera de una identificación demasiado rígida entre el país y el Hinduísmo. Hay que recordar que en la India colonial una parte muy notable de la población era musulmana y la separación de Pakistán debe entenderse también en este contexto. Así, para Gandhi resultaba más útil una noción universalista de espiritualidad que fuera compatible con un estado laico, capaz de acoger la diversidad religiosa de la región en un único espiritualismo nacional.

En el caso de Rabindranath Tagore, si bien el poeta se alejaba de la versión más nacionalista del espiritualismo (despreciaba la "idolatría de la nación") se ha terminado convirtiendo en una figura sustantiva en la internacionalización del vínculo que funde India y espiritualidad, como otros intelectuales orientales 
formados en Reino Unido. $Y$ precisamente su influencia en otros autores se ha visto como el germen de la expansión del espiritualismo político a su versión pan-asiática. El japonés y amigo de Tagore, Kakuzo Okakura, formado en Oxford y en su orientalismo metropolitano, viajó a India e principios del siglo XX y escribió en 1903 "Ideals of the East" (escrita originalmente en inglés). La obra se convertiría pronto en un hito fundamental del espiritualismo pan-asiático. El poeta y también amigo de Tagore, Yoneuo Noguchi, ya justificaba en sus cartas de 1938 la idea de "Asia para los asiáticos" en su justificación del imperialismo japonés (en el contexto de la invasión de Japón a China en 1938). La dimensión espiritual que los japoneses esgrimieron para justificar su invasión fue un acicate más al rechazo de la Revolución Cultural por las cuestiones espirituales, sin embargo, el caso de la espiritualidad en China representa una variación reciente y especialmente interesante de la emergencia de este concepto contemporáneo de espiritualidad universal que nace en las entretelas de la estrategia política.

Con la victoria del comunismo chino en 1949, el materialismo se convirtió en la ideología oficial del estado y las cuestiones religiosas (incluida la espiritualidad) se declararon a vestigios de un pasado feudal que debía ser superado, empezando por la eliminación de sus bienes, sus representantes y todo lo demás. Sin embargo, esa noción de espiritualidad universal, que se reclama oriental al mismo tiempo que es una occidentalización, conservó su significado político en dos enclaves singulares, como son Singapur y Taiwan. Desde ahí, hervideros del pensamiento político regional, ya en los años noventa del siglo $X X$, los intelectuales chinos han empezado a reclamar (con cierto consentimiento del estado) la espiritualidad neo-confuciana como "espíritu de la nación" china y también como contribución cultural de china al humanismo global (Tu and Tucker 2003, 2004). La rentabilidad del turismo espiritual, de la exportación de objetos e ideologías vinculadas a la espiritualidad y de las empresas relacionadas con el yoga, el taichi, el qi gong y otras actividades relacionadas con ello, han contribuido en gran medida a que las autoridades chinas hagan la vista gorda a la identificación entre espiritualidad y Oriente, demostrando de paso que tal identificación no es tanto un hecho histórico como un producto estratégico de las vicisitudes políticas y económicas de las relaciones internacionales. En palabras de Van der Veer:

Estos nuevos usos políticos de la espiritualidad ahora se consideran New Age o una forma de despolitización. Sin embargo, (...) estas formas de entender la espiritualidad como apolítica o incluso antipolíticas, oscurecen el hecho de que la espiritualidad, al igual que la secularización, han sido empleadas en luchas radicales tanto en Oriente como en Occidente. (Van der Veer 2009:1117-8)

\subsection{EL POTENCIAL HUMANO Y LA SUBJETIVIZACIÓN}

Teniendo en cuenta las fuentes más plenamente modernas de la espiritualidad, así como su asociación general con las cuestiones del bienestar/malestar, aún queda por reseñar justamente el elemento clave que sirve de eslabón entre todos los elementos anteriores y, por ende, de cemento conceptual y moral 
para la espiritualidad terapéutica contemporánea. Este cemento capaz de unir todas las piezas anteriores y formar un único producto lo encontramos en el llamado Movimiento del Potencial Humano, en su cosmovisión, en su ética, en su mística y en su terapéutica. De acuerdo con Michael York (2003:95), el Movimiento del Potencial Humano es una convergencia difusa de corrientes en la que se mezclan grupos, individuos, ideologías y técnicas de carácter tan diferente como el Zen y el Análisis Transaccional, la Cienciología/Dianética y el Reiki, o la Meditación Trascendental y la Gestalt. Aunque el movimiento se inspira en autores como Aldoux Huxley o Alan Watts, se suele considerar iniciadores de la corriente a Abraham Maslow, a Roberto Assagioli y a Fritz Perls, y su lugar de referencia es el Instituto Esalen, un lugar de estudio y retiro espiritual fundado en 1963 en el sur de California ${ }^{3}$. Lo característico del movimiento y de las muchas corrientes ideológicas y terapéuticas que lo cruzan es la idea seminal del humanismo de Maslow: el énfasis en la autorrealización y el crecimiento personal, un discurso que chocaba con el rígido ambiente moral de los años cincuenta del siglo XX.

Como ha enfatizado Elisabeth Puttick (2000), el movimiento se originó en los años sesenta como una rebelión contra la psicología, contra la religión y contra la moral de la época. La rebelión incluía una dimensión milenarista (la llamada de la Era de Acuario) que hablaba de la transformación del mundo y de la conciencia, a lo que el movimiento se refería como "un cambio de paradigma". La rigidez y represión de los cincuenta, en la que la mayoría de los visitantes de Esalen habían crecido, fue retratada por Wilhelm Rich en 1968 como la causa de neurosis, de desórdenes psicosomáticos y de problemas sociopolíticos como el fascismo. Así, frente a los rigores de la disciplina, el deber, la firmeza, el trabajo duro y la frugalidad, el movimiento se propuso trabajar sobre las "virtudes blandas" como la expresión espontánea del afecto. Así, en un intento de superar las limitaciones del psicoanálisis y del conductismo, Maslow desarrolló una psicología especialmente optimista, que partía de la jerarquía malinowskiana de las necesidades humanas y proponía un enfoque orientado a la superación de las limitaciones personas. En contraste con los antecesores, su psicología se basaba más en la salud mental que en las patologías, y desde ahí se daba una especial importancia a las elecciones individuales, los valores y las creencias. Su principal contribución teórica fue precisamente el concepto de "autorrealización", basado en la premisa de que, habiendo resuelto las necesidades materiales y emocionales, el ser humano estará completamente pleno y será plenamente dueño de sí mismo cuando pueda dedicarse a cultivar la espiritualidad, concebida aquí como "experiencias cumbre" de tipo extático. Sus postulados se alinean así con el fundamento teórico del individualismo. Más allá de las cuestiones teóricas, su impacto terapéutico es enorme, entre otras cosas por la variedad aun creciente de técnicas derivadas de su enfoque. Las teorías de Maslow se adaptarían y evolucionarían en numerosas escuelas humanísticas, pero las más relevantes quizá hayan sido la de Carl Rogers, centrada en el counselling (el asesoramiento centrado en la persona), la Gestalt de Fritz Perls, el Análisis Transaccional de Eric Berne o los Grupos de Encuentro de Will Schutz. El punto en común de todos ellos es la exploración de los sentimientos y las

\footnotetext{
${ }^{3}$ Un repaso exhaustivo de los pensadores más influyentes en el Movimiento del Potencial Humano y en la New Age en general lo podemos encontrar más detallado en Prat (2012:25-63)
} 
relaciones. En la medida en que esta área de la vida se consideraba una parcela íntima y tabú en la sociedad de su tiempo, cabe atribuirle a Maslow también el mérito de haber comenzado el proceso de des-estigmatización cultural y social de la psicoterapia. $Y$ en este proceso se da lugar también a lo que Hanegraaf ha llamado la psicologización de la espiritualidad y la sacralización de la psicología (Hanegraaff 1998:224).

El viraje del Movimiento del Potencial Humano hacia la espiritualización ya se produce en los años setenta. De acuerdo con Puttik (2000), existía alrededor de Esalen la sensación de que el cambio había comenzado pero no se había dado una transformación tan radical como la que se esperaba, y ello se relacionó inmediatamente con la espiritualidad. Este giro al principio planteaba problemas porque se percibía una contradicción entre el significado convencional del término (asociado al sufrimiento y la mortificación en el Cristianismo) y la meta terapéutica de la autorrealización característica de Maslow. Esta contradicción también traía consigo una tensión característica que se ha mantenido en el tiempo, la tensión entre un enfoque centrado en el individuo, la auto-aceptación y el amor a uno mismo, por un lado, y la ideología del amor y el servicio a otros. En esta tensión, la noción de espiritualidad se asimilaba originalmente con el sacrificio del yo en pos del deber o de los demás, de manera que se hizo necesario actualizar la noción de espiritualidad para superar en la medida de lo posible esta tensión y dar lugar con ello a una espiritualidad terapéutica, positiva, centrada en el desarrollo personal y hasta cierto punto expresión misma de la autorrealización.

En este giro conceptual, tuvo una influencia determinante el orientalismo, que en el caso del Movimiento del Potencial Humano no era una influencia local, derivada de los grupos orientales radicados en los Estados Unidos, sino más bien una influencia derivada de Jung y el Psicoanálisis (Fromm y Maslow establecieron algunas conexiones teóricas entre el psicoanálisis y el budismo zen, aunque la meditación y la psicoterapia no se percibieron como complementarias antes de los años setenta). Así por ejemplo, Fitzgerald (1986:186) argumenta que Alan Watts, el introductor del taoísmo en Estados Unidos, estaba en realidad más próximo a la psicoterapia que al misticismo en su particular interpretación de las tradiciones místicas orientales. Y a partir de aquí también se puede percibir una convergencia recíproca. En los setenta, se hacen populares los aún hoy más conocidos movimientos religiosos de raigambre oriental, que se introducen como adaptaciones de las técnicas de meditación, y muy en particular, adaptaciones a la forma de vida occidental, ajetreada pero confortable. De este modo, la meditación se independiza de los monasterios y se intenta introducir entre las rutinas cotidianas y el bienestar material. En la medida en que los gurús indios no tenían conexiones con las tradiciones culturales y espirituales de Occidente, la psicología humanista sirvió de puente y herramienta para el diálogo y la expansión. Así, los terapeutas del movimiento del Potencial Humano empezaron a usar la meditación como técnica complementaria del crecimiento personal y experimentaron con sus grupos, especialmente en Esalen. Con el tiempo, la transición de la terapia (o de la salud) a la práctica espiritual (sanación) se vio como una continuación lógica acorde con las teorías maslowianas de la autorrealización. 
Un buen ejemplo de esta convergencia se refleja especialmente bien la doctrina de Osho (Bhagwan Shree Rajneesh), quien conoció el Movimiento del Potencial Humano en la India, pues entre sus discípulos occidentales se encontraban algunos miembros del movimiento, y entre cuyas múltiples técnicas se encuentra una versión mística de la catarsis de Reich (la meditación activa). La fusión entre un marco doctrinal propio del tantrismo y la psicoterapia a estas alturas resultaba relativamente fácil. No obstante, a pesar de estas convergencias, cabe señalar que Osho y otras fusiones de misticismo y psicoterapia han sido criticadas abundantemente tanto por terapeutas como por los partidarios de tradiciones budistas e hinduistas más convencionales, entre otras cosas por una paradoja sustantiva entre las metas espirituales de las religiones dhármicas (la extinción del yo) y el énfasis en el yo del Movimiento del Potencial Humano. Al mismo tiempo, el éxito de estas fusiones se extendió a otras formas religiosas, como el chamanismo y los paganismos contemporáneos, en los que los valores del crecimiento personal y la espiritualidad como terapia han transformado (en algunos casos de forma muy significativa) la interpretación de las fuentes originales y el sentido de las prácticas tradicionales.

\section{LA ILUSIÓN DE LA ESPIRITUALIDAD APOLÍTICA}

Si no te encuentras bien, no hables de ello, a menos que quieras más malestar. Si escuchas hablar a los demás de su enfermedad, añades energía a su enfermedad. Rhonda Byrne.

Para terminar, una de las características más prominentes de la transición de la espiritualidad desde los tormentos corporales a la ética del bienestar es la aparente despolitización de la noción de espiritualidad. Los defensores de una espiritualidad apolítica suelen encontrarse entre los partidarios más radicales de la dicotomía entre espiritualidad y religión. Como ha mostrado Mathew Woods (2009), la asimilación de lo religioso con lo institucional, con las iglesias históricas y sus prácticas de adoctrinamiento, la espiritualidad contemporánea es reivindicada ahora como un rechazo a la dimensión política de la religiosidad, pero en el día a día de la expansión New Age es fácilmente apreciable una dimensión política actual de las espiritualidades terapéuticas. Para empezar, esta dimensión política se encuentra en las propias formas organizativas de los individuos, grupos y redes del ambiente holístico, en el que se reproducen relaciones de poder herederas de las relaciones de género y hegemonía que se dan en el resto de esferas de la vida del individuo espiritual (Cornejo 2012, Fedele y Knibe 2013). Pero además, como ha señalado Eva Illouz (2010) en La salvación del alma moderna: la cultura terapéutica de la autoayuda, la espiritualidad terapéutica contemporánea se ha constituido como el reservorio ideológico y de sentidos del capitalismo, penetrando con fluidez en las políticas empresariales, para las que la meditación (especialmente en la forma del mindfulnes) resulta una propuesta eficaz (rentable) frente a los problemas de estrés y los síndromes de burned out de los trabajadores (en detrimento de las mejoras en las condiciones laborales). 
En una dirección similar apunta Vanina Papalini (2013) al afirmar que:

En términos de la acción social, de la movilización colectiva o de los reclamos sindicales, este tipo de discurso tiene un efecto desarticulador. Los años 90 son un momento de consolidación de la retórica de la autoayuda y de generalización de una cultura terapéutica que resulta condición necesaria para un modelo sociopolítico neoprudencialista, que delega la obligación de autocontrolarse y autosostenerse en el propio sujeto. (Papalini 2013:171)

En su Selling Spirituality. The silent takeover of religión, Jeremy Carrete y Richard King (2005) nos muestran cómo el pensamiento New Age encaja especialmente bien en los círculos empresariales porque es ecléctico y flexible, porque su énfasis sobre el desarrollo personal concuerda con el auge de las soft skills como el liderazgo, la intuición, la visión, etc. y además, la idea de la transformación personal (central en el pensamiento New Age) encaja tam bien con las necesidades empresariales de adaptar la fuerza de trabajo a las demandas cambiantes del mercado. De ahí que estos autores lleguen a afirmar que "la espiritualidad se ha convertido en una Religión Genéticamente Modificada, el aditivo alimentario que hace el neoliberalismo más agradable al paladar" (Carrete y King, 2005:132). Frente a interpretaciones como éstas, el antropólogo Paul Heelas, ha reivindicado el rol crítico típicamente romántico que presenta la New Age como religión subjetiva y subjetivizante, cuyo fin precisamente es criticar las formas religiosas altamente institucionalizadas. Así, en Spiritualities of Life, New Age Romanticism and Consumptive Capitalism (2009), Heelas contesta las críticas contra las espiritualidades terapéuticas argumentando que ni siempre ni necesariamente estamos ante un comportamiento mercantil o mercantilizante cuando hablamos de las terapias alternativas. No obstante, el propio Heelas reconoce algunos elementos ideológicos que han tenido y continúan teniendo una cierta centralidad en el liberalismo primero y en el neoliberalismo después, como ideologías propias de las diferentes etapas del capitalismo. En este sentido, Heelas encuentra determinante el peso de una "búsqueda del yo", propia del romanticismo y de lo que Charles Taylor ha llamado la ética de la autenticidad.

Frente a la exaltación romántica de la subjetividad, sin embargo, Barbara Ehrenreich (2009) ha denunciado la tiranía del individualismo sobre el propio individuo. En su libro Bright sided (titulado en su edición en español como "Sonríe o muere"), Ehrenreich empieza describiendo su proceso personal en la superación de un cáncer de mama, durante el que se enfrentó, entre otras cosas, a la dureza de un optimismo totalitario por parte de la comunidad terapéutica. En este medio, el sufrimiento, el dolor, la ira o las incertidumbres se rechazan y no se permite fácilmente su expresión. Antes al contrario, se puede llegar a imponer la tortuosa idea de que la enfermedad es un regalo del destino o del Universo para enseñarnos valiosas lecciones de crecimiento personal y elevación espiritual, por lo que el enfermo debe sentirse agradecido. A la vista de tales distorsiones, Ehrenreich concluye mostrándonos las vueltas de tuerca mediante las que la espiritualidad contemporánea sigue vinculada con sus avatares modernos y premodernos. 
Una de las mejores cosas que se pueden decir del pensamiento positivo es que consiguió erigirse en alternativa al calvinismo, una de las peores es que acabó manteniendo algunos de los rasgos calvinistas más tóxicos: la forma despiadada de juzgar, similar a la condena del pecado que hacía la religión, y la insistencia en hacer una constante labor de autoexamen. La alternativa norteamericana al calvinismo no iba a ser el hedonismo, ni siquiera la defensa de las emociones espontáneas, no. Para el que piensa en positivo, las emociones siguen siendo sospechosas, y uno debe pasarse el día supervisando atentamente su propia vida interior. (Ehrenreich 2009:108)

\section{REFERENCIAS}

ÁLVAREZ LÁZARO, Pedro (1985). Masonería y librepensamiento en la España de la Restauración:(Aproximación histórica). Universidad Pontificia Comillas. Madrid.

ASAD, Talal, (2003). Formations of the secular: Christianity, Islam, modernity. Stanford University Press.

ASAD, Talal, (2009). Genealogies of religion: Discipline and reasons of power in Christianity and Islam. JHU Press.

BASTIDE, Roger, (1961) Sociología y psicoanálisis, Compañía General Fabril Editora, Buenos Aires.

BASTIDE, Roger, (1983), Sociología de las enfermedades mentales. Siglo XXI, Madrid.

BESANT, Anie Wood (2011) Annie Besant: An autobiopraphy, Cambridge University Press, Cambridge.

BOFF, Leonardo (1981). Pasión de Cristo: pasión del mundo:(hechos, interpretaciones y significado ayer y hoy). Sal terrae. Santander.

BORREGUERO, Eva (2004). Hindú: Nacionalismo religioso y política en la India contemporánea (Vol. 174). Los libros de la Catarata.

CARRETTE, Jeremy R., KING, Richard (2005). Selling spirituality: The silent takeover of religion. Routledge, London.

CHRISTIAN, William A (1997) Las apariciones de Ezquioga. Ariel. Barcelona.

COMAROFF, Jean, COMAROFF, John L., (1991). Of revelation and revolution, volume 1: Christianity, colonialism, and consciousness in South Africa. Chicago: University of Chicago Press.

CONSTANTINIDES, Pamela. III at ease and sick at heart: Symbolic behaviour in a Sudanese healing cult, en Lewis, Ioan (ed.), Symbols and sentiments, 1977, p. 61-84.

CORNEJO, Monica (2001). "El debate actual sobre pentecostalismo". Política y Sociedad, 37, 151-160.

CORNEJO, Mónica 2012. "Religión y espiritualidad ¿dos modelos enfrentados? Trayectorias post-católicas entre budistas Soka Gakkai", en Revista Internacional de Sociología 70(2):327-346.

DE HEUSCH, Luc (1973), Estructura y praxis, Siglo XXI, Madrid.

DE MARTINO, Ernesto (1999). La tierra del remordimiento. Bellaterra. Barcelona. 
DE MOTES BERNET, Jordi Maluquer. El socialismo en España, 1833-1868. Editorial Crítica, 1977.

DONATI, Pierpaolo. (1994). Manual de sociología de la salud. Ediciones Díaz de Santos. Madrid.

EHRENREICH, Barbara. Bright-sided: How the relentless promotion of positive thinking has undermined America. Macmillan, 2009.

FEDELE, Anna Fedele; KNIBE, Kim 2013. Gender and Power in Contemporary Spirituality. Etnographic Approaches. London: Routledge.

FITZGERALD, Frances, (1986) Cities on a Hill. Simon \& Schuster. New York.

FLANAGAN, Kieran; JUPP, Peter C. (eds.) (2007) A sociology of Spirituality: Ashgate Publishing Limited. Aldershot.

FORMAN, Robert K. (2004). Grassroots spirituality. Imprint Academic. Exeter. GEERTZ, Clifford (1995). La interpretación de las culturas, Gedisa. Barcelona. GEORGE, L. K., LARSON, D. B., KOENIG, H. G., McCULLOUGH, M. E. (2000) "Spirituality and health: What we know, what we need to know", in Journal of social and clinical psychology, 19(1), 102-116.

GIORDAN, Giovanni (2007) "Spirituality: from a religious concept to a sociological theory", en Flanagan, Kieran; Jupp, Peter C. (eds.) (2007) A sociology of Spirituality: Ashgate Publishing Limited. Aldershot. Pp. 161-80.

GOWER, Christopher (2006) Hablar de sanación ante el sufrimiento, Desclée de Brouwer, Bilbao.

HALL, Daniel E., Meador, Keith G., Koenig, Harold G. (2008). "Measuring religiousness in health research: Review and critique". Journal of religion and health, 47(2), 134-163.

HANEGRAAFF, Wouter (1998) New age Religion and Western Culture, Brill. Leiden.

HEELAS, Paul (2009). Spiritualities of life: New age romanticism and consumptive capitalism. John Wiley \& Sons, London.

HEELAS, Paul, WOODHEAD, Linda (2008) The Spiritual Revolution. Why religion is giving way to spirituality, Blackwell. Oxford.

HORTA, Gerard (2001). De la mística a les barricades: introducció a l'espiritisme català del XIX dins el context ocultista europeu. Edicions Proa, Barcelona.

ILLOUZ, Eva 2010. La salvación del alma moderna. Terapia, emociones y la cultura de la autoayuda. Barcelona, Katz.

JUAN PABLO II (1995). Evangelium vitae. Buenos Aires.

KOENIG, Harold (2012) "Why do research on spirituality and health, and what do the results mean?" Journal of Religion and Health, 51, 460-467.

KOENIG, Harold G. (2008) "Concerns about measuring "spirituality" in research", The Journal of nervous and mental disease, 196(5):349-355.

KOENIG, Harold, KING, Dana, CARSON, Verna B. (2012) Handbook of religion and health. Oxford University Press, Oxford.

LEVIN, Jeff (2009). Restoring the spiritual: Reflections on arrogance and myopia-allopathic and holistic. Journal of religion and health, 48(4), 482-495.

LEVIN, Jeffrey S.; TAYLOR, Robert Joseph; CHATTERS, Linda M. (1994) "Race and gender differences in religiosity among older adults: Findings from four national surveys". Journal of Gerontology, vol. 49(3), S137-S145.

LÉVI-STRAUSS, Claude, (1995) Antropología Estructural. Paidós. Barcelona.

LEWIS, Ioan M. (1971) Ecstatic Religion. Penguin Books. Harmondworth, London. 
LISÓN TOLOSANA, Carmelo (2004). La España mental 2: el problema del mal (Vol. 2). Ediciones Akal, Madrid

LOWIE, Robert, (1990) Religiones Primitivas, Alianza Editorial, Madrid.

LUJAMBIO, Alonso. Retratos de familia: un dramaturgo liberal, un historiador católico y un espiritista maderista. Ediciones Arkhe., 2011.

McGUIRE, Meredicth (1998) Ritual Healing in Suburban America. The State University. Rutdgers.

METRAUX, Alfred (1955) "La comedie rituelle dans la possession", en Diogène $11: 26$

OBEYESEKERE, Gananath, (1985). Depression, Buddhism, and the work of culture in Sri Lanka. Culture and depression, 134-152.

OBEYESEKERE, Gananath, (1990). The work of culture: symbolic transformation in psychoanalysis and anthropology. University of Chicago Press. Chicago.

PABLO VI (1968). Humanae vitae. Ediciones Paulinas. Santiago.

PAPALINI, Vanina (2013) "Recetas para sobrevivir a las exigencias del neocapitalismo (o de , cómo la autoayuda se volvió parte de nuestro sentido común)", en Nueva Sociedad, 245, 163-177.

PARK, Nan Sook, S., LEE, B. S., SUN, F., KLEMMACK, D. L., ROFF, L. L., \& KOENIG, H. G. (2013). Typologies of religiousness/spirituality: Implications for health and well-being. Journal of religion and health, 52(3), 828-839.

PRAT, Joan (2012). "Hacia una arqueología de los nuevos imaginarios culturales". En Gazeta de Antropología, 28 (3).

PUTTIK, Elisabeth (2002) "Personal Development: the Spiritua- lisation and Secularisation of the Human Potential Movement", en Sutcliffe, Steven, Bowman, Marion 2000. Beyond New Age: exploring alternative spirituality. Edinburgo: Edinburgh University Press.

QUINCENO, J. Margarita, VINACCIA, Stefano (2009) "La salud en el marco de la psicología de la religión y la espiritualidad". Diversitas, 5(2):321-336.

Roof, Wade Clark (2003). Religion and Spirituality. Handbook of the Sociology of Religion, 137.

SHUMAN, Joel James, MEADOR, Keith G. (2003). Heal thyself: Spirituality, medicine, and the distortion of Christianity. Oxford University Press. Oxford.

VAN DER VEER, P. (2009). Spirituality in modern society. Social Research: An International Quarterly, 76(4), 1097-1120.

WOOD, Mathew (2009) "The nonformative elements of religious life: Questioning the Sociology of Spirituality Paradigm", Social Compass 56, 2, 23748.

YORK, Michael 2004. Historical Dictionary of New Age Movements. Oxford: Scarecrow Press. 